\title{
Creating noisy 3D shapes - an implementation using free and open source tools
}

\author{
Zoltán Derzsi (zd8@nyu.edu) and Robert Volcic (robert.volcic@nyu.edu) \\ New York University Abu Dhabi
}

\begin{abstract}
Distorting the shape of a three-dimensional object in a randomised manner is a very difficult task. We present a script in this paper that programmatically generates spheres with added radius noise, utilising Meshlab's software back-end. We successfully tested the script on Mac OS, Windows, and Linux operating systems. We also have given instructions on how to set up the environment for each platform, and how to replicate what the script does manually.
\end{abstract}

\section{Introduction}

For years, vision scientists have been investigating shape perception [Koenderink and Doorn, 1992] [Todd, 2004] [Doerschner et al, 2011]. Creating reliable noisy stimuli is a non-trivial task, especially when 3D stimuli are required. Such stimuli are not only useful for vision science, but also for haptics [Hu et al, 1999] [Phillips et al, 2009] [Norman et al, 2012] [Norman et al, 2016] and robotics [Miller et al, 2003]. A method of generating 3D objects was published by Flip Phillips [Phillips, 2004], where a method was described to distort a 3D object with noise, by moving surfaces or points of the object along their surface normal vectors. We found that it is particularly difficult making a surface displacement map using conventional tools. For example, when the creation of an object with a randomised displacement map based on a sphere is required, its mesh is defined as a flat 2D array. A dynamic change of spatial frequency is required to form a displacement map with a constant spatial noise bandwidth across all directions when the 2D array is used as the object mesh.

We found that dedicated 3D editor software products (proprietary: Renderman, 3D Studio Max, Cinema 4D, free an open source: Blender, Meshlab) can do this, but scripting these packages to generate an arbitrary number of randomised objects is very often difficult or impossible. To the best of our knowledge, at the time of writing, we are not aware of a software product that can produce truly randomised objects programmatically.

Meshlab [Cignoni et al, 2008] is particularly interesting, as it allows the manipulation of objects through filters defined in a markup language-style interface. The script accompanying this document runs in Matlab and Octave, and it can programmatically generate the filter files, which it then promptly executes on the included sphere using Meshlab's command-line interface. 


\section{Implementation}

A proposed model of this approach is also described in great detail in David Ebert's 'Texturing and Modeling: a Procedural Approach' textbook. The script described here generates a noisy object from a sphere by randomly dislocating a surface along its normal vector. The surface displacement maps are generated with fractals, and the user can specify the following parameters:

- The spatial frequency bandwidth in octaves

- The amplitude of the noise, normalised to the size of the object

- Optionally, the seed number of the fractal generator algorithm

The script executes Meshlab, and automates the following procedure:

1., Generate a sphere of the desired radius, or load the model to be randomised.

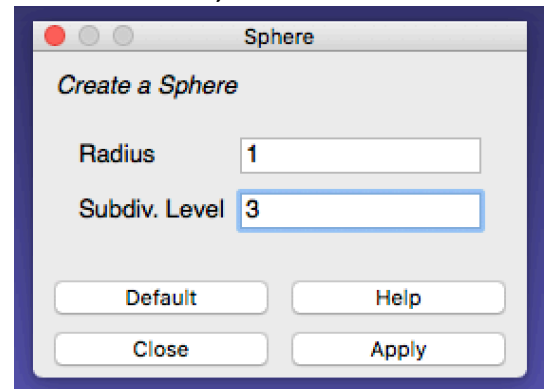

Figure 1: Generating the sphere

- Go to [Filters] -> [Create New Mesh Layer] -> [Sphere]

- Set the desired diameter and detail level (for example, 'Subdiv. Level $=6$ ' for highdetail object), and then click [Apply]

2., Randomise the mesh. 


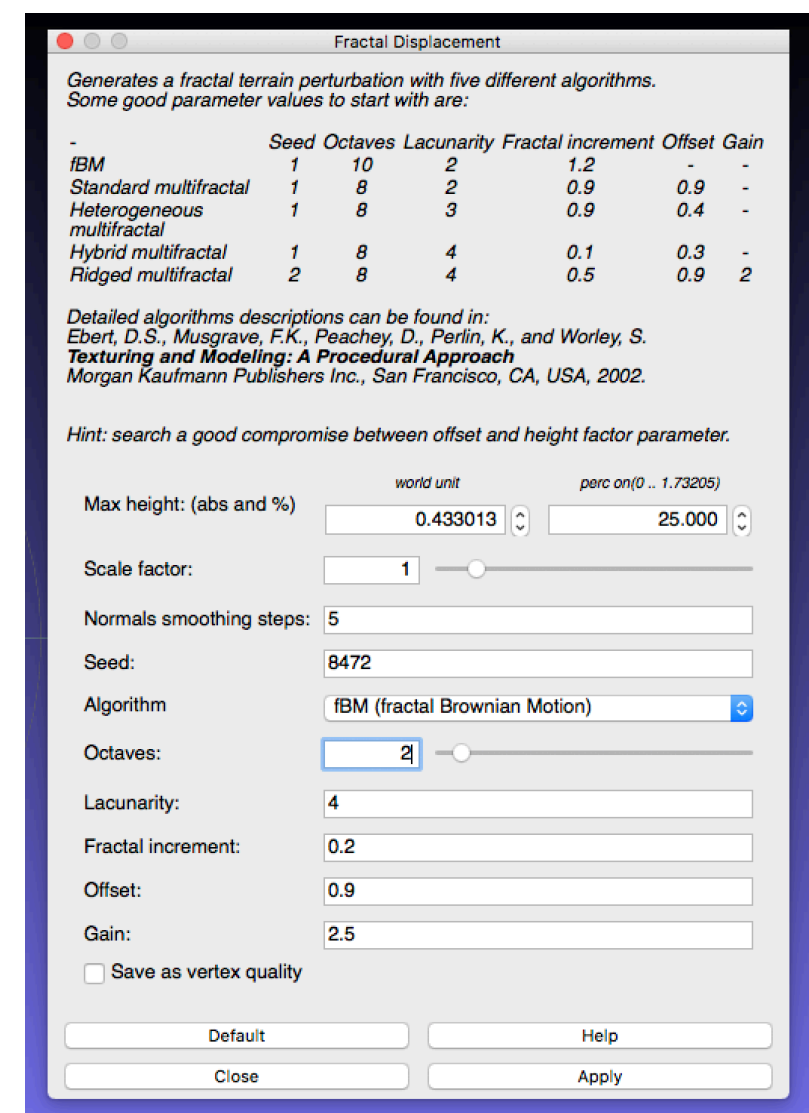

Figure 2: Randomising the object with fractals

- Go to [Filters] -> [Smoothing, Fairing and Deformation] -> [Fractal Displacement]

- Select the algorithm to be 'fBM (fractal Brownian Motion)'

- Specify the 'Max height' of the object, in percentage (for example, 25\%)

- Specify 'Octaves' of the spatial noise (a typical value is 2)

- Optionally, specify the seed of the algorithm

- Press [Apply] to generate the randomised object

The output should look similar to this: 


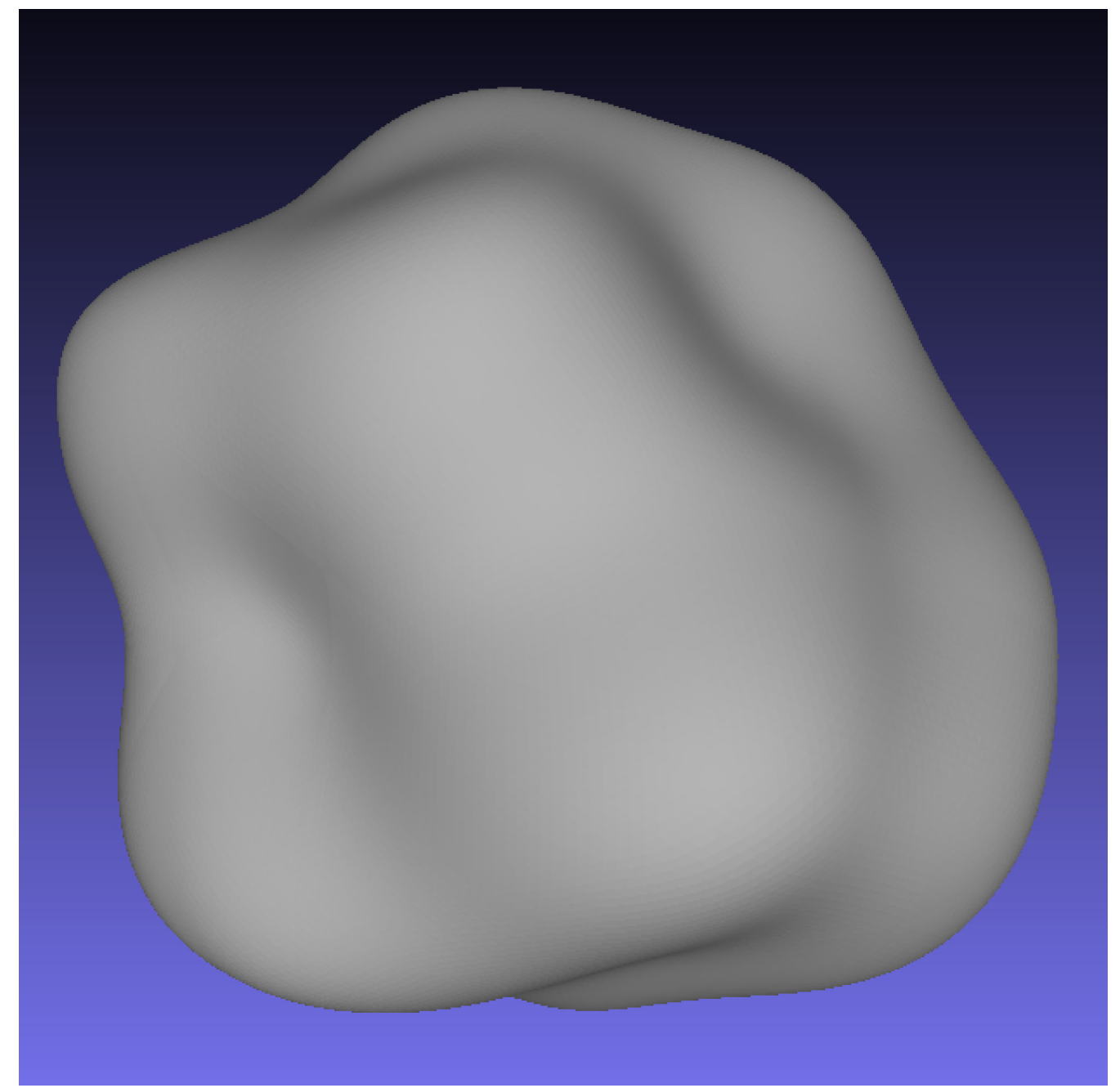

Figure 3: An example output with $25 \%$ noise amplitude and 2 octaves bandwidth

There is a sphere VRML file included in the script bundle's 'sphere' directory, but the same technique can be applied to any 3D object that can be loaded into Meshlab. By running generate_object_catalogue.m, the two main parameters can be adjusted: the number of octaves to be used in the filtering ('octaves $=. .$. ), and the noise amplitude relative to the object's radius, in percent ('amplitudes $=.$. '). If many octaves and amplitudes are specified, the script will generate every possible iteration.

The script then calls 'open_config_file()', 'randomise_surface()', and 'close_config_file()' to generate the filter file for Meshlab. Then, the script assembles the correct combination of command line arguments, and calls 'meshlabserver' in the specified path. The filter files are left in the 'temp' directory, and the output 'stl' files containing the noisy objects are in the 'output' directory.

Please note that in the filter configuration file, the maximum height of the object must be defined. We have included some sample values for spheres at various radii in the 'randomise_surface()' function. However, if other models are used, not changing this value will lead disproportionate noise amplitudes. This parameter can be obtained from a filter file that has been created using Meshlab, manually, as follows:

1., Generate a sphere of the desired radius, or load the model to be randomised. 
Go to [Filters] -> [Create New Mesh Layer] -> [Sphere]

Set the desired diameter and detail level, and then click [Apply]

2., Randomise the mesh.

Go to [Filters] -> [Smoothing, Fairing and Deformation] -> [Fractal Displacement]

Select the desired spatial frequency bandwidth in octaves. Also, enter the desired noise amplitude on 'Max height: (abs and \%)' as a percentage in the 'perc on (...)' field, and the software will calculate the required parameter in its internal world units.

\section{Platform differences}

While we developed this script on a Mac (macOS 10.12), and used Meshlab 1.3.3 along with Matlab R2016b, we also made sure that the same script works on other platforms with minimal modification. The script was tested on Octave 3.8.0 as well. However, to get the backend of Meshlab to work, additional work-arounds are required.

Please note that due to the nature of software development, some features might be different in subsequent versions, which might be originated from Meshlab, or due to version differences in the same libraries used in different operating systems. This script was developed and used in February 2017.

For each platform, the work-arounds are listed below for the default installation paths of Meshlab.

We have placed an error message in line 18 of generate_object_catalogue.m, and added the three default meshlabserver paths for the three plaforms. Please comment out the error message, and the corresponding path for the platform used. Also, please have a look at the low-level documentation included in the code.

Mac

On a Mac, please use Meshlab 1.3.3. The latest '2016' version for Mac OS does not work with this script.

In generate_object_catalogue.m, the meshlabserver executable by default is:

meshlabserver_executable = '/Applications/meshlab.app/Contents/Mac0S/meshlabserver';

If, after the execution of the script, nothing appears in the output directory, and Matlab's console shows the output depicted on figure 1, it is possible that the external Qt libraries are not found by meshlabserver. Make sure that the open-source Qt libraries are properly installed on the system, by downloading and installing it from https://www.qt.io/download/. 


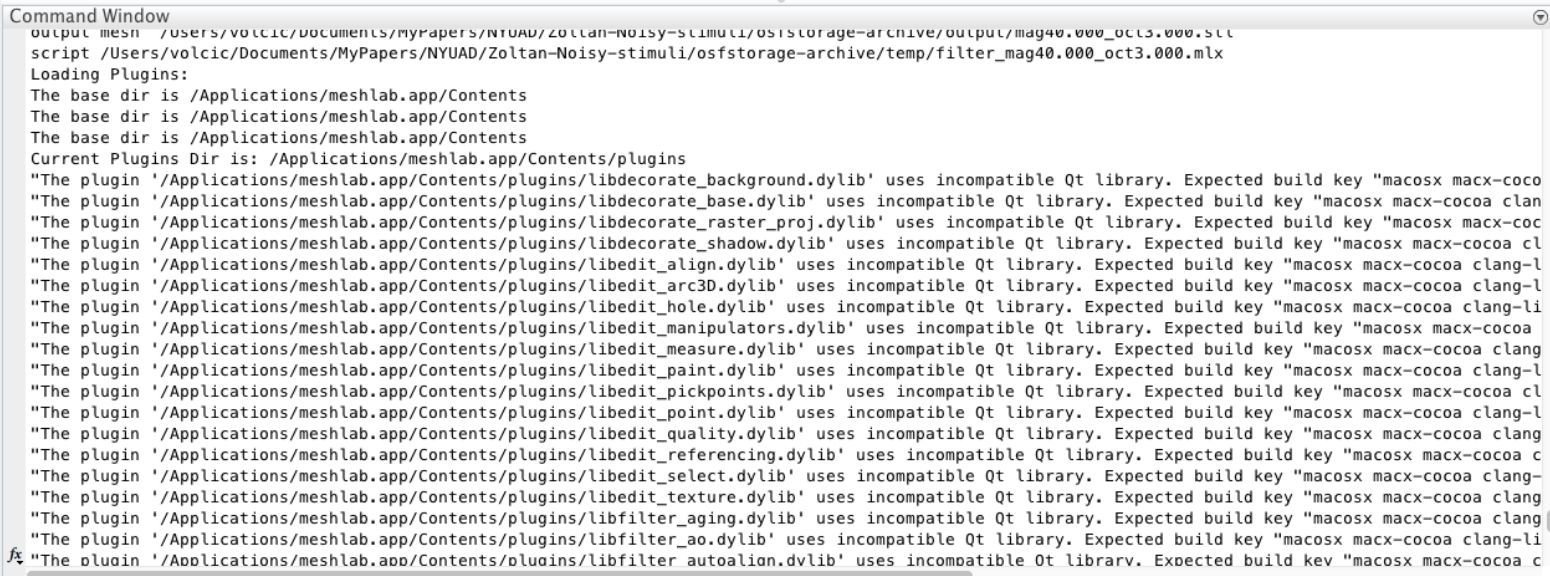

Figure 4: If the script does not generate the objects and the 'uses incompatible Qt library' error message appears in Matlab's command window, please install the Qt libraries .

Linux

We tested the script on a computer with the 64-bit version of Ubuntu 17.04, and installed Meshlab from the repositories, by typing in a terminal:

$\$$ sudo apt-get install meshlab

In generate_object_catalogue.m, the meshlabserver executable is in the path:

meshlabserver executable = 'meshlabserver'

\section{Windows}

We tested the script on a computer which ran the 64-bit Windows 10 operating system, with an NVIDIA graphics card. Please note that Meshlab does not run without OpenGL drivers installed and supported hardware (particularly, it won't run on computers with Intel's graphics accelerators)

On Windows, the latest (2016) version of Meshlab works. In generate_object_catalogue.m, the meshlabserver executable needs to be specified as follows:

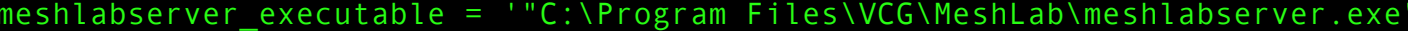

Please note that the extra quotation marks are needed to account for the space in the path. 


\section{Summary}

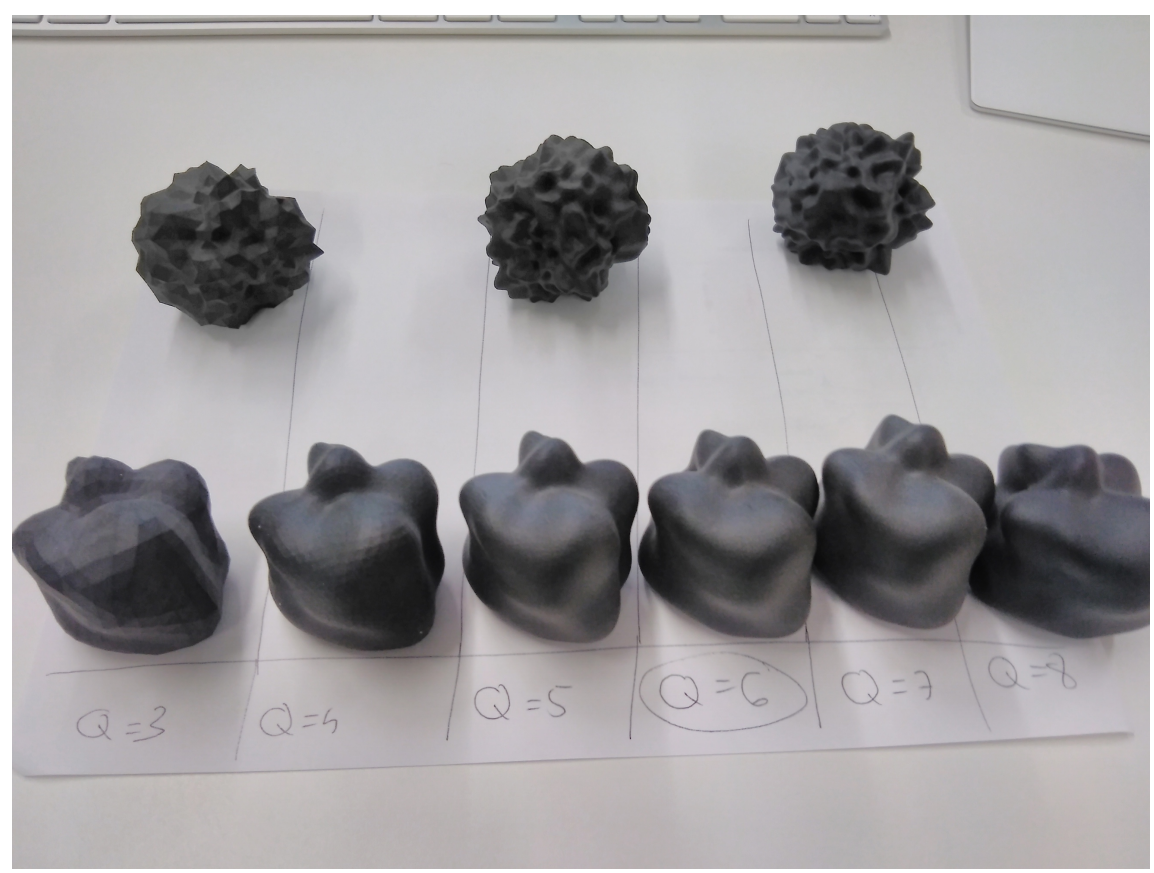

Figure 5: A set of example randomised objects that have been generated with the script and were 3D printed using laser sintering. Top row: 3 octaves of spatial noise, bottom row: 2 octaves of spatial noise. From left to right, the same model is rendered with an increasing number of polygons.

We have presented a Matlab/Octave script that allows the generation of truly randomised objects, which can work with any imported model and can be used to produce randomised objects without the need for manual interaction.

\section{Acknowledgements}

We would like to thank Ivan Camponogara for helping with cross-platform testing.

\section{References}

Koenderink, J. J., \& Van Doorn, A. J. (1992). Surface shape and curvature scales. Image and vision computing, 10(8), 557-564.

Todd, J. T. (2004). The visual perception of 3D shape. Trends in cognitive sciences, 8(3), 115121.

Doerschner, K., Fleming, R. W., Yilmaz, O., Schrater, P. R., Hartung, B., \& Kersten, D. (2011). Visual motion and the perception of surface material. Current Biology, 21(23), 2010-2016.

Hu, Y., Eagleson, R., \& Goodale, M. A. (1999). Human visual servoing for reaching and grasping: The role of 3D geometric features. In Robotics and Automation, 1999. Proceedings. 1999 IEEE International Conference on (Vol. 4, pp. 3209-3216). IEEE. 
Phillips, F., Egan, E. J. L., \& Perry, B. N. (2009). Perceptual equivalence between vision and touch is complexity dependent. Acta psychologica, 132(3), 259-266.

Norman, J. F., Phillips, F., Holmin, J. S., Norman, H. F., Beers, A. M., Boswell, A. M., ... \& Ronning, C. (2012). Solid shape discrimination from vision and haptics: Natural objects (Capsicum annuum) and Gibson's "feelies". Experimental brain research, 222(3), 321-332.

Norman, J. F., Phillips, F., Cheeseman, J. R., Thomason, K. E., Ronning, C., Behari, K., ... \& Lamirande, D. (2016). Perceiving object shape from specular highlight deformation, boundary contour deformation, and active haptic manipulation. PloS one, 11(2), e0149058.

Miller, A. T., Knoop, S., Christensen, H. I., \& Allen, P. K. (2003, September). Automatic grasp planning using shape primitives. In Robotics and Automation, 2003. Proceedings. ICRA'03. IEEE International Conference on(Vol. 2, pp. 1824-1829). IEEE.

Phillips, F. (2004). Creating noisy stimuli. Perception, 33(7), 837-854.

Cignoni, P., Callieri, M., Corsini, M., Dellepiane, M., Ganovelli, F., \& Ranzuglia, G. (2008, July). Meshlab: an open-source mesh processing tool. In Eurographics Italian Chapter Conference (Vol. 2008, pp. 129-136).

Ebert, D. S. (2003). Texturing \& modeling: a procedural approach. Morgan Kaufmann. 